\title{
La paradoja de la salud mental en Colombia: entre los derechos humanos, la primacía de lo administrativo y el estigma
}

\author{
Paradox of Mental Health in Colombia: Between Human Rights, the Predominance of the Administrative \\ and the Stigma \\ O paradoxo da saúde mental na Colômbia: entre direitos humanos, a primazia do administrativo e o estigma
}

Dora María Hernández Holguín ${ }^{\text {a }}$

Universidad de Antioquia, Colombia

doram.hernandez@udea.edu.co

ORCID: http://orcid.org/0000-0002-1050-6625

Cristian Felipe Sanmartín-Rueda

Universidad de Antioquia, Colombia

ORCID: http://orcid.org/0000-0002-6810-1057
DOI: https://doi.org/10.11144/Javeriana.rgps17-35.psmc Redalyc: http://www.redalyc.org/articulo.oa?id=54557477003

Fecha de recepción: 24 Octubre 2017

Fecha de aprobación: 17 Mayo 2018

Fecha de publicación: 01 Noviembre 2018

\section{Resumen:}

En las últimas décadas se viene promoviendo en el mundo la reforma de sistemas de salud mental hacia servicios de base comunitaria e integrados en los sistemas locales de salud. Al respecto, se han reportado logros en algunos países latinoamericanos y del Caribe, mas no así en Colombia. Sobresalen en el mundo las brechas en la atención y la confrontación entre derechos humanos-criterios económicos, para la prestación de servicios en salud mental. Se hizo una investigación con enfoque histórico-hermenéutico en la que participaron 23 profesionales de la salud mental en entrevistas y un grupo de discusión; se realizó análisis categorial de la información. Se halló cómo los derechos humanos, la rentabilidad financiera, la calidad de los servicios y el estigma conforman directrices que orientan, de manera contradictoria, la salud mental en Colombia. Se discutió sobre la conveniencia de realizar un análisis ético y político de la política pública de salud mental en el país.

Palabras clave: salud mental, sistemas de salud, política pública, legislación sanitaria, gestión en salud, Colombia.

\section{Abstract:}

In the last decades a reform to the mental health system is being promoted all over the world towards community-based services integrated to the local health systems. Some Latin-American and Caribbean countries have reported achievements on this issue, but not Colombia. The gaps in the health care are noticeable worldwide as well as the confrontation between human rights and financial criteria for the provision of health services. A research was carried out based on a historical-hermeneutic approach; 23 mental health professionals took part in it through interviews and discussion groups. A categorial analysis of the gathered information was carried out. It showed how the human rights, financial cost-effectiveness, service quality and the stigma form the guidelines that drive, in a contradictory way, the mental health services in Colombia. A discussion is carried out on the convenience to develop an ethical and political analysis of the mental health public policies in our country.

Keywords: mental health, health system, public policy, public health laws, health management, Colombia.

\section{Resumo:}

Nas últimas décadas tem se promovido no mundo tudo a reforma de sistemas de saúde mental para serviços de base comunitária e integrados nos sistemas locais de saúde. A esse respeito, tem relatórios de sucesso em alguns países latino-americanos e do Caribe, mas na Colômbia não. Destaca-se no mundo a lacuna no atendimento e o confronto entre direitos humanos e critérios económicos, para a prestação de serviços de saúde mental. Uma pesquisa com abordagem histórico-hermenêutica foi feita, na que participaram 23 profissionais de saúde mental em entrevistas e um grupo de discussão; realizou-se análise categorial da informação. Achou-se que os direitos humanos, a rentabilidade financeira, a qualidade dos serviços e a estigma conformam diretrizes orientando, de maneira contraditória, a saúde mental na Colômbia. Foi debatida a conveniência de realizar análise ética e política da política pública de saúde mental no país.

Palavras-chave: saúde mental, sistemas de saúde, política pública, normativa sanitária, gestão da saúde, Colômbia.

\footnotetext{
a Autora de correspondencia: doram.hernandez@udea.edu.co
} 


\section{Introducción}

La Organización Mundial de la Salud (OMS, en su informe sobre la salud en el mundo, dedicado en el año 2001 a la salud mental, anunciaba cómo los sistemas de salud mental en muchos países estaban siendo objeto de grandes reformas, entre ellas la desinstitucionalización de las personas con trastorno mental, el desarrollo de programas basados en la comunidad y la integración en el sistema de salud general (1. Reformas que la OMS ya venía fomentando desde 1990, a partir de la Declaración de Caracas (2 y con su llamado a direccionar los servicios de salud mental hacia servicios de base comunitaria, integrados en la atención primaria y en los sistemas locales de salud.

Pasados 15 años, y a manera de evaluación de la reforma de los servicios de salud mental que se promovió desde la Declaración de Caracas, sobresalieron en América Latina y el Caribe las propuestas innovadoras y logros por parte de Belice, con su red de servicios ambulatorios; Brasil, con su modelo de atención psicosocial; Chile, con los resultados de un programa nacional de salud mental $(3,4.5)$; y Cuba, con sus centros comunitarios de salud mental (3). Estos países, más allá de casos territoriales han logrado avanzar sistemáticamente en la implementación de una política o plan nacional de salud mental conforme a sus condiciones sociales, culturales, políticas y económicas.

La experiencia de Brasil es un ejemplo cercano sobre cómo se fueron dando tensiones y transformaciones en el campo de la salud mental en el ámbito nacional, específicamente desde su reforma del Sistema Único de Salud, que conllevó una reforma psiquiátrica y con ella el funcionamiento de los centros de atención psicosocial (CAPS). En este caso, se cuestionaron como principios en salud mental: (a) el método epistémico de la psiquiatría, centrado en las ciencias naturales; (b) el concepto de enfermedad mental como error, sinrazón o peligrosidad; (c) el aislamiento y la institución asilar como recursos terapéuticos; y (d) el tratamiento moral presente en las terapias normalizadoras (6). Cabe aclarar que este ejemplo se refiere a reflexiones que cuestionan la salud mental con respecto a la lógica asilar y manicomial como práctica central ante diagnósticos psiquiátricos — reflexión pertinente para la realidad de ese país- y que lleva a preguntarse cuáles serían los principios propios para Colombia.

En el estudio de la transformación de los servicios de salud mental brasileños se propende por un cuestionamiento epistemológico y ético, en el sentido de que realmente se modifiquen las prácticas en salud mental; por ejemplo, en relación con "la locura" debe ponerse en discusión, además de su significado, las estrategias que se aplican, y revisar si puede llegar a ser posible la superación del modelo tradicional de servicios en salud mental. Para ello, se necesita un enfoque histórico que permita deconstruir y con ello comprender las formas de reproducción o transformación del modelo psiquiátrico (6).

La misma OMS, aunque centrada aún en la enfermedad mental, sigue invitando a miradas que contemplen los determinantes estructurales de la salud y los derechos humanos, como lo hace en el plan de acción integral sobre salud mental 2013-2020 (7, cuyos principios y enfoques transversales defienden, entre otros: los derechos humanos; la práctica basada en la evidencia, no solo científica sino en prácticas óptimas que tengan en cuenta consideraciones de carácter cultural; el enfoque multisectorial, que incluya salud, educación, empleo, vivienda y otros sectores necesarios; la emancipación de las personas con trastornos mentales y discapacidades psicosociales y su participación en la promoción, las políticas, la planificación, la legislación, la prestación de servicios y la investigación en materia de salud mental.

De otro lado, si bien el hecho de centrar la salud mental en los trastornos mentales no es en sí mismo algo negativo cuando estas manifestaciones tienen una base biológica, sí se ha cuestionado el énfasis económico de sus desarrollos, en términos de inversión y de costos para las personas que usan los servicios, quienes encuentran barreras de acceso a los beneficios de estos avances de tecnologías en salud (8, 9). Para mostrar la brecha entre el acceso a servicios por afecciones de salud mental con respecto al acceso por salud física, se ha llamado la atención sobre cómo en países con altos ingresos, el 33\% de las personas con trastorno 
recibe tratamiento, frente al $75 \%$ que lo recibe en caso de diabetes, y, peor aún, solo el $2 \%$ de las personas con las mismas condiciones neuropsiquiátricas recibe tratamiento en países de bajos y medianos ingresos (5).

De hecho, suena contradictorio cuando se afirma que el enfoque comunitario de la salud mental se fundamenta en los derechos humanos y en el trabajo intersectorial y a la vez se le justifica con el argumento de que las formas alternativas de tratamiento comunitario son más costoeficaces que aquellas ofrecidas en el ámbito hospitalario (1), lo que muestra directrices paradójicas entre lo que promueven y la práctica, pues las decisiones en salud mental se pueden ver enfrentadas así a criterios económicos versus derechos humanos, que pueden llegar a ser contradictorios y excluyentes en su aplicación.

En Colombia, la salud mental es particularmente compleja, pues guarda una estrecha relación con adversidades sociohistóricas en cuanto a políticas, inequidades y desastres, tanto naturales como por violencia $(10,11)$. A esto se suma a que no se ha encontrado que la literatura disponible resalte acciones del país en el sentido comunitario propuesto para el mundo; más bien se le reconoce como un país con una fuerte tendencia al estudio desde la psiquiatría y las neurociencias de los mecanismos neurofi siológicos, genéticos y comportamentales de los trastornos mentales y cuyo sistema de salud, de manera explícita, prioriza la rentabilidad de los servicios privados sobre los servicios públicos de salud, mas no como un país con avances importantes en la salud mental poblacional $(4,12)$.

Como documentos oficiales que direccionan el quehacer en salud mental en Colombia, se cuenta, en primer lugar, con la Política Nacional de Salud Mental (PNSM) (13), aprobada por medio de la Resolución 2358 de 1998, que contempla como objetivos "promover la salud mental en el país y prevenir la aparición de la enfermedad mental; mejorar el acceso, cobertura y calidad de la atención en salud mental en todas sus fases"; y, en segundo lugar, la Ley 1616 o Ley de Salud Mental, del 21 de enero de 2013 (14), de acuerdo con la cual:

La salud mental es de interés y prioridad nacional, es un derecho fundamental, es tema prioritario de salud pública, es un bien de interés público y es componente esencial del bienestar general y del mejoramiento de la calidad de vida de colombianos y colombianas.

Con la iniciativa del Ministerio de Salud y Protección Social, la PNSM ha tenido varios intentos fallidos de actualización. El primero, en el 2007, liderado por la Asociación Colombiana de Psiquiatría, con su propuesta de "Política nacional del campo de la salud mental, documento-propuesta para discusión y acuerdos" (15). El segundo intento, en cumplimiento de la Ley 1616, se presenta en el "Documento propuesta de ajuste de la PNSM para Colombia”, del 2014 (16), el cual plantea como dificultades en el proceso de implementación de la política vigente desde 1998, el cambio de gobierno nacional, modificaciones constantes de la normatividad en salud, falta de decisión política, problemas con la disponibilidad de recursos financieros y escasa divulgación.

Ante esta situación, se hace necesario un mayor acercamiento al sistema de salud mental en el país, no precisamente a partir de la normatividad vigente y los informes técnicos que se han producido, sino a través de la cotidianidad de quienes viven el sistema desde diferentes roles laborales de tipo asistencial, administrativo, científico, directivo, legal, político y docente. Esto permitirá una mayor comprensión sobre la realidad en la implementación de las directrices nacionales en salud mental y orientar preguntas de investigación para el futuro sobre el sistema y las políticas de salud mental.

En síntesis, la Ley de Salud Mental no se ha reglamentado y la PNSM no se ha implementado ni se ha actualizado. Ante este panorama, surgió la inquietud sobre la forma como funciona el sistema de salud mental en Colombia, desde la experiencia y la perspectiva de profesionales del campo de la salud mental.

\section{Materiales y métodos}

Se hizo una investigación naturalista, con enfoque histórico hermenéutico, dado el interés en la interpretación de los valores, la comprensión del hombre como sujeto y de sus experiencias en el mundo vital cotidiano (17). Participaron en total 23 profesionales, abogados, médicos y psicólogos, con posgrado en administración, 
salud pública, psicología clínica y psiquiatría, ocho de ellos profesionales con experiencia laboral de por lo menos cinco años en diversos ámbitos de la salud mental, en equipos profesionales de tipo docente, asistencial, administrativo, científico, directivo, legal y político, y con población en diversas etapas del ciclo evolutivo. Fueron invitados a entrevista individual como experto en legislación (EL), experto en administración de servicios (EAS) o experto científico (EC). Además, se tuvieron en cuenta los aportes que sobre el tema hizo una mesa de discusión sobre talento humano en salud mental, organizada por el Grupo de Salud mental de Antioquia, Colombia, en la cual participaron 15 profesionales con perfiles similares a los participantes entrevistados.

Las entrevistas fueron semiestructuradas en la modalidad de entrevista a expertos (18), cuya guía fue sometida previamente a un estudio exploratorio para su afinación, tanto en el contenido como en la forma de las preguntas. Los expertos fueron contactados en las instituciones a las cuales se encontraban vinculados; luego del consentimiento informado para su participación en el estudio, se realizaron las entrevistas, seis de manera presencial y dos virtuales, las cuales fueron grabadas y transcritas; posteriormente, se codificaron hasta hallar las categorías sobre el fenómeno en estudio (19). El muestreo fue teórico, en el sentido de que la recolección de datos fue guiada por los conceptos derivados en el proceso (19). La mesa de discusión fue grabada, transcrita y analizada por medio de codificación selectiva, y sus aportes se expresaron en la complementación a las categorías de análisis que para ese momento ya se habían identificado.

En todo el proceso se tuvieron en cuenta los aspectos éticos, según los lineamientos de la Resolución 8430 de 1993 del Ministerio de Salud de Colombia (20) y los criterios de la Declaración de Helsinki de la Asociación Médica Mundial, del 2008 (21). En coherencia con ellos, se cumplió con el consentimiento informado por parte de los expertos participantes, a quienes se explicó la naturaleza, la duración y los fines de la investigación; el método y los medios con los que se llevaría a cabo; los inconvenientes y los riesgos esperados, y se les garantizó el anonimato, en caso de que lo consideraran conveniente. El estudio contó con el aval del Comité de Ética de la Investigación de la Facultad Nacional de Salud Pública.

En los resultados se desarrolla la categoría "los derechos humanos, la rentabilidad financiera, la calidad de los servicios y el estigma social, enfoques que orientan contradictoriamente la salud mental en Colombia”.

\section{Resultados}

En esta sección se exponen algunas interpretaciones realizadas y soportadas en testimonios que dan cuenta de la situación de la salud mental en el sistema de salud colombiano, así como el estigma que desde este y la sociedad se presenta respecto a esta dimensión de la salud.

\section{Los derechos humanos, la rentabilidad financiera, la calidad de los servicios y el estigma. Directrices que orientan de manera contradictoria la salud mental en Colombia}

\section{Los derechos humanos y las fallas del sistema de salud}

Se encontró, desde la perspectiva de los participantes, que en Colombia no hay un enfoque definido en la dirección del sistema de salud mental, sino un cúmulo de directrices, evidentes, implícitas y contradictorias que hacen presencia de diferentes formas. De manera explícita, los derechos humanos son intencionados a partir de la Constitución Política y desde allí se espera sean dirigidos a la normatividad. Por otra parte, la búsqueda de rentabilidad financiera al servicio del interés particular puede verse también en la legislación y en el funcionamiento del sistema de salud. La calidad de los servicios está presente en los protocolos de atención y en los sistemas de gestión de calidad de las instituciones de salud, en tanto que el estigma a la salud mental es 
evidente en la normatividad, en los servicios de salud y en la sociedad en general, y afecta tanto a las personas que solicitan los servicios como a sus familias y al talento humano en salud.

Si se busca una relación lógica entre estos, puede decirse que los lineamientos dados con base en los derechos humanos y en la calidad de los servicios van en pro de una oferta en defensa de la vida y la calidad de vida de las personas que acuden a los servicios de salud mental. Sin embargo, el mayor desarrollo se ha orientado hacia la calidad de los procesos administrativos y financieros, cuya implementación en muchas ocasiones no tiene en cuenta a la persona que solicita el servicio, y por su parte, los derechos humanos, enunciados en la Constitución Política y en la normatividad en general, en la práctica quedan supeditados al cumplimiento de requisitos administrativos que en su intención defienden intereses económicos particulares.

Si bien las luchas sociales en salud, enunciadas por algunos de los expertos, han podido poner en evidencia las grandes falencias del sistema de salud -e. g., en el caso de la salud mental se logró que la Corte Constitucional se refiriera a esta dimensión de la salud como constitucional-, son muchas las barreras de acceso a servicios de calidad debido a problemas estructurales y socioculturales que orientan las decisiones administrativas en los servicios de salud, no con base en los derechos humanos, sino inclusive en contra de ellos y a favor de beneficios económicos. De esta manera, la "salud de la población" se subordina a lo administrativo.

Los expertos consideran que hay un vacío en la legislación nacional en salud en temas de salud mental, principalmente en la Ley 100 de 1993 (22) como pilar del Sistema General de Seguridad Social en Salud (SGSSS). Apenas en las reformas de la Ley 1122 de 2007 (23) y en la Ley 1438 de 2011 (24), 14 y 18 años después de la aprobación de Ley 100, respectivamente, se incluyó la atención, tanto por entidades públicas como por privadas, y se hizo énfasis en la atención de niños, niñas y adolescentes. En el 2013 se aprobó la Ley de Salud Mental, sin reglamentar aún, y a pesar de los avances en la legislación todavía son graves los problemas que existen.

Para los participantes, la defensa del derecho a la salud mental en la ejecutoria del SGSSS se ha convertido en un asunto personal, además de profesional. Desde esta posición, ven claramente en el funcionamiento del sistema la presencia contradictoria de directrices: los derechos humanos, la rentabilidad financiera, la calidad de los servicios y el estigma en la administración de la salud mental en Colombia (figura 1) y como estas directrices se evidencian en falencias de tipo estructural, conceptual y sociocultural del SGSSS con respecto a la salud mental.

\section{Enfoques que orientan la salud mental en Colombia}

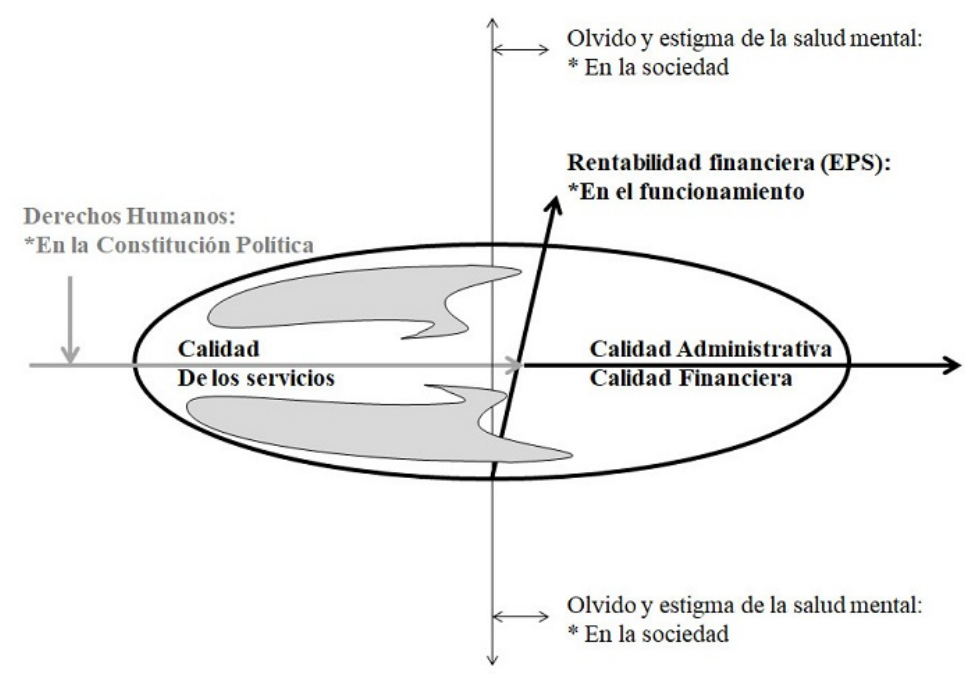

FIGURA 1

Paradojas en las directrices de salud mental en Colombia.

Elaboración propia. 


\section{Los derechos humanos terminan supeditados a los aspectos administrativos}

Para los expertos, es claro que "la Ley 1616 está basada en los derechos humanos" (EL) y que en ella hay un esfuerzo por superar las falencias del sistema de salud:

La ley 1616 no enfrenta el Sistema General de Seguridad Social en Salud pero tiene en cuenta las falencias de este (EAS).

Sin embargo, a pesar de la referida ley y de la concepción de la salud mental como derecho desde la Constitución, los expertos hacen referencia a fallas estructurales, conceptuales y socioculturales (figura 2) que impiden el logro de la salud mental como derecho.

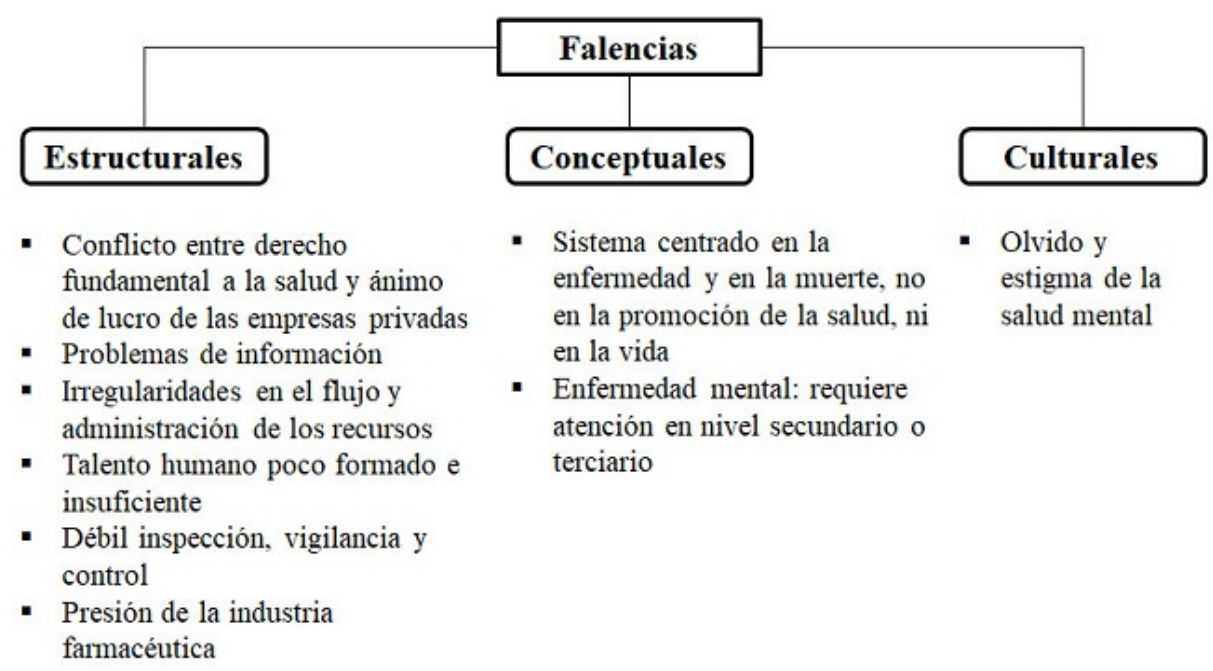

FIGURA 2

Falencias del Sistema General de Seguridad Social en Salud - Mental de Colombia.

Elaboración propia.

Los expertos afirman, puntualmente, que el sistema no tiene problemas de presupuesto:

Las problemáticas para el acceso a servicios de salud mental están generadas por otros factores, no porque no estén los recursos financieros. El principal problema es la falta de recurso humano, de infraestructura y el gasto de bolsillo de los usuarios. (EAS)

Otro experto se refiere a las fallas del sistema como estructurales:

La falla estructural más grave del sistema es su propia esencia, es que tiene una confrontación entre el derecho fundamental de la salud y el ánimo de lucro de las empresas privadas. El sector privado ha trabajado con la lógica de empresa y libertad de mercado, con barreras de contención de costos, barreras administrativas, económicas, de acceso, es la misma lógica: bajos costos para tener mayor ganancia. Otra falla es la falta de información oportuna; otra es el flujo de los recursos y fallas en los servicios, en las redes de servicios de salud mental (EL).

Con respecto a la conceptualización en salud mental en el sistema, otros expertos afirman:

La concepción de trastorno mental en el sistema de salud es como si fuera una enfermedad únicamente para tratarla en segundo o tercer nivel de atención, y como no hay suficiente recurso humano para atenderla en el nivel primario, entonces los problemas se cronifican. (EC)

Tenemos un problema de conceptos, pues tenemos un sistema centrado en la enfermedad y en la muerte y no en la promoción y la prevención. (EC)

Según los participantes, la forma de concebir la salud mental hace que esta sea invisible en los niveles primarios de atención, pero es de resaltar que los expertos cercanos a procesos de atención y rehabilitación también vean fallas importantes en estos niveles de atención para los diversos problemas de salud mental: 
La rehabilitación en trastornos mentales no existe, por lo tanto la discapacidad no se disminuye, a menos que exista la interdisciplinariedad; es necesario mayor claridad sobre la rehabilitación, la cual no se considera rentable. (EC)

En el tema de sustancias psicoactivas hay una incipiente cobertura [...] Por un lado por su costo-efectividad, que en el mejor de los casos logra el 30\% de rehabilitación, la atención en procesos de recaídas son altamente costosos y especializados, por esto no hay acceso efectivo a los servicios aunque estén en el plan de beneficios. (EAS)

Los procedimientos, actividades e intervenciones que requiere una persona víctima de la violencia intrafamiliar conllevan fuertes barreras de acceso para la autorización de algunos procedimientos, hacen que esa problemática no sea atendida adecuadamente y se le suma también el problema de la capacidad técnica del recurso humano disponible que también es insuficiente en número para atender esta problemática. (EAS)

Lo anterior deja ver más aún el problema como generalizado, que incluye una concepción errada de la salud mental y la defensa fácilmente visible de la sostenibilidad financiera, como se expresa en las siguientes palabras:

Hay un artículo en la Ley 1438, que dice que las decisiones que se tomen en salud deben estar acordes a la sostenibilidad financiera del sistema. Por lo tanto, si yo soy médico o profesional y tengo que tomar una decisión tendría que pensar en eso en vez de pensar en el beneficio del paciente. (EL)

Siguiendo con las fallas enunciadas:

[...] Una falla grande son los medicamentos [...] porque obviamente las multinacionales tienen un interés muy diferente a la salud pública. De hecho, solo están haciendo investigaciones en lo que les produce; otra falla es la vigilancia y control, no tienen la infraestructura ni personal capacitado para ello [...] la mayoría de información en la que ellos basan sus decisiones es información de las mismas EPS [Empresas Promotoras de salud] [...] ellas mismas deciden qué información dan y cuando la envían. (EC)

Sobre la manera cómo afecta esta falla en el caso de tratamiento de adicciones, otro experto dice:

Encontramos múltiples instituciones de garaje para el tratamiento en adicciones [...] Los estándares, es decir, la norma existe, pero hay una gran debilidad de las funciones de inspección, vigilancia y control de parte del Estado hacia estos centros que no acogen los mecanismos de habilitación. (EAS)

Otra falla es la participación comunitaria, casi que desapareció y se confundió con algo que se llama participación comercial, es decir, el call center, la queja, pero no la participación. (EL)

Con respecto a la atención en salud mental de niños, niñas y adolescentes, afirman:

Los niños, niñas y adolescentes desde la Constitución Nacional [igual que en muchas normas, protocolos y guías de prácticas clínicas] se dice que son poblaciones que deben ser no solamente preferentes, sino diferentes a los demás. Lo que a mí me ha tocado es que los niños y adolescentes, que son especialmente protegidos, son los que hemos tenido que tutelar [...] porque la entidad no los trata así como pide la ley, porque no existen programas especializados [...] por ejemplo en una de las tutelas, el muchacho tenía una esquizofrenia, dependía de su papá por ser discapacitado y se muere el papá y le dice la entidad que no le sigan dando los medicamentos hasta que le salga la pensión. (EL)

Otro ejemplo se da en un departamento de más de 40 municipios, los expertos dicen:

No tenemos servicios hospitalarios de psiquiatría infantil. (EAS)

¿Cuáles son las necesidades en salud mental de niños y adolescentes? [...] Yo creo que muchas [...] o sea todas! No hay atención ¿sí? O sea no hay las posibilidades de atención, no hay el personal lo suficientemente formado, no hay la disponibilidad de recurso $[\ldots](\mathrm{EC})$

Sin embargo, los derechos humanos no se violentan solamente a los usuarios, también al personal de salud que presta sus servicios. Para uno de los expertos: 
Al tiempo que los servicios de salud mental carecen de profesionales, muchos de ellos están desempleados. Es frecuente la contratación con intermediación laboral, inestabilidad, bajos salarios, demoras en el pago, y cargas laborales excesivas. (EAS)

Contrariamente a la brecha que señalan los expertos — entre lo que enuncia la normatividad sobre la salud mental y la atención real - sí hay coincidencia y lógica entre la búsqueda de calidad de procedimientos administrativos y la rentabilidad financiera, que también defiende la normatividad. Es así como en el funcionamiento del sistema de salud mental se enfrentan la necesidad de atención de los usuarios y la primacía de los intereses particulares por medio de la rentabilidad financiera.

Como se puede ver en las siguientes citas, se refieren, por ejemplo: contratos cortos y mal remunerados; concentración de personal especializado en las grandes ciudades; tiempo dedicado a multiplicidad de registros, al servicio de trámites administrativos.

En Colombia se hacen marcados esfuerzos por contar con servicios de calidad, sin embargo, la calidad se ha centrado en lo administrativo y lo financiero, no en los servicios.

La mayoría de nuestros municipios solo cuentan con una psicóloga adscrita a la oficina local de salud para tareas administrativas y de vigilancia en salud pública, y a veces una en la comisaría de familia. Esta psicóloga, suele estar nombrada entre uno y tres meses al año [...] En muchos casos pueden pasar hasta tres psicólogas o psicólogos por año en el mismo municipio, pero se van por la mala remuneración y la excesiva carga laboral. (EAS)

Hay departamentos completos donde no se cuenta con especialistas en psiquiatría. Más del 50\% de los psiquiatras del país están en una sola ciudad que es Bogotá [...] (EC)

$\mathrm{Al}$ profesional le toca mucho registro y le queda poco tiempo para el paciente y como lo miden es por el registro, se dedican más al registro que al paciente. (EAS)

\section{Estigma de la salud mental}

Lo anterior también coincide con el olvido y el estigma de la salud mental, no solamente en el sector salud, sino como característica sociocultural que se da en la familia, en la comunidad, en la sociedad y, lo que es peor, en los órganos legislativos. Los expertos se refirieron al estigma que ha acompañado a la salud mental y a su manifestación por medio de los trastornos mentales a lo largo de la historia:

Hay un legado cultural, prehistórico, de prejuicio a la salud mental, del que nadie se ha escapado [...] ni ningún país [...] ni ninguna cultura. (EAS)

Estigma hacia el talento humano y su quehacer:

La confusión de roles también refleja el estigma y la subvaloración, en la exigencia de ejercer roles distintos al campo disciplinar, al psicólogo se le pide hacer actividades de trabajador social, o se confunde al psicólogo social con el clínico. (EAS, EC)

Las recomendaciones de la investigación sobre salud mental del municipio las dimos en una reunión con una persona del equipo que nos iba a recibir por su propio interés, no de la entidad. (EC)

Tambien se resaltó en el proceso de formación y en el ejercicio profesional:

En una investigación, los estudiantes de medicina en Colombia, en comparación con estudiantes de una ciudad de otro país, veían el dedicarse al área de salud mental como algo menos científico, como un área de cierta especulación. (EAS)

No me explico cómo algunos llegamos a ser psiquiatras [...] todo está en contra de eso, inclusive la formación. (EAS)

En una consulta compartida entre psiquiatra y médica general, mientras la psiquiatra hacía la anamnesis del paciente la médica se estaba pintando las uñas. (EAS) 
De manera específica, también se refirieron a los "conceptos hipermorales y punitivos" que rodean el tema de consumo de drogas, que además dejan de lado la posibilidad de que haya "consumo sano", o las ideas de "machismo" que pueden afectar el tema de violencia intrafamiliar y abuso sexual y que atraviesan a toda la sociedad, incluyendo los servicios.

Para finalizar, hay opiniones encontradas entre los expertos con respecto a las expectativas en relación con el tema. Algunos consideran que a pesar de las dificultades, se están logrando y se pueden lograr cambios positivos, y otros, menos optimistas, piensan que se requiere, para poder hacer los cambios necesarios, el empoderamiento de la sociedad civil, un concepto concertado de salud y de salud mental y un pacto social por la salud que incluya también a los académicos, de manera que los conocimientos se complementen y se pongan al servicio del sistema.

\section{Discusión}

Como pudo verse en los resultados anteriores, el sistema de salud mental en Colombia funciona de manera contradictoria, en una tensión permanente entre los derechos humanos, la rentabilidad financiera, la calidad de los servicios y el estigma, los cuales se expresan en falencias de tipo estructural, conceptual y sociocultural. Una estructura centrada en lo económico y en franca contradicción con los derechos humanos que predica la norma, concepciones al servicio de lo económico, que se centran en la enfermedad, en tecnología de alto costo y mínimo acceso, y que dejan de lado la expresión positiva, relacional y colectiva de la salud mental en la cotidianidad. Por último, desde el estigma y el olvido, no se ha reconocido el lugar relevante que ha desempeñado la salud mental en el bienestar de las personas y comunidades en Colombia.

Como punto de partida, se puede afirmar que la intención técnica y ética de quienes han liderado la Ley de Salud Mental y la PNSM y sus actualizaciones ha fracasado, a pesar de los esfuerzos que han hecho, y que se requiere un análisis profundo de la política pública de salud mental en el país, en su sentido ético y político y en consonancia con la realidad sociocultural en la que opera. Para el análisis propuesto de la "política pública de salud mental en Colombia”, esta puede ser entendida, en general, en los términos de Roth: política pública traduce el concepto inglés de public policy, entendiendo por este el proceso de elaboración y puesta en marcha de programas de acción pública por parte de autoridades gubernamentales (25). Y en palabras de Lahera (2006):

Una política pública de excelencia corresponde a aquellos cursos de acción y flujos de información relacionados con un objetivo político definido en forma democrática, que son desarrollados por el sector público y, frecuentemente, con la participación de la comunidad y el sector privado. Debe, por ello, incluir orientaciones o contenidos, instrumentos o mecanismos, definiciones o modificaciones institucionales, así como la previsión de sus resultados [...] Las políticas públicas de excelencia incluyen el aspecto político como su origen, objetivo, justificación o explicación pública, y si no son enmarcadas en un amplio proceso de participación pueden sesgar a los actores públicos: los especialistas hacia la tecnocracia y los comunicadores o encuestólogos hacia al populismo inmediatista. (26, p. 77 )

De acuerdo con lo anterior, podría decirse que la gestión de la política de salud mental en Colombia se ha visto afectada, en parte, por una debilidad en la participación social, y con ello en sus bases políticas, debido a que en los logros alcanzados no quedan representados ni defendidos los diversos sectores de la sociedad; al menos no todos aquellos con un real interés en la salud y en la salud mental. Es posible que se logren representaciones parciales y que, en este sentido, se corra siempre el riesgo de hacer esta gestión desde la "tecnocracia" o desde el "populismo inmediatista", sin lograr un impacto en la salud mental de las poblaciones.

El análisis de política propuesto debería centrarse en sus aspectos éticos y políticos más que en aquellos técnicos, pues en la experiencia relatada se insinúa una tensión constante entre los expertos técnicos y 
quienes a partir de un poder político dan viabilidad o no a la propuesta de una nueva política, lo que podría explicar la paradoja mencionada entre los elementos identificados con base en lo referido por los participantes.

Para Deborah Stone (27), las implicaciones éticas de las políticas se deben a que sus objetivos pueden organizarse alrededor de cuatro conceptos: equidad, que corresponde a la búsqueda de justicia por parte de la comunidad y con base en la cual busca responderse: ¿quién recibe qué, cuándo y cómo?; eficiencia, es decir, la comunidad busca obtener el máximo provecho de un insumo o lograr un objetivo al menor costo; seguridad, busca responder a necesidades de integrantes de la comunidad, definir quién necesita, qué necesita y quién provee; y libertad, en ella se privilegia la libertad colectiva, aunque se tengan que restringir las individuales. A estos conceptos, Roth (25) agrega el de justicia y en su conjunto los presenta como aquellos que justifican las políticas públicas para todo Estado o autoridad pública moderna, y considera que los derechos humanos expresan, desarrollan y buscan estos mismos objetivos.

Vale la pena, en esta invitación a un análisis de la política pública de salud mental en Colombia, tener en cuenta los aportes de Pedro Medellín (28) sobre la centralidad del régimen político y el gobierno en los procesos de estructuración de las políticas públicas. En este análisis, los elementos claves que permiten diferenciar los regímenes políticos son la territorialidad e institucionalización del orden. La primera en relación, entre otras cosas, con la cohesión interna, del Estado con la sociedad y los mercados, cuyos problemas se reflejan en el poder político para el control territorial. Y la institucionalización del orden, en relación con la capacidad de los principios y los valores institucionales para mantener la unidad en el poder político, el valor y la estabilidad de las estructuras, funciones y procedimientos de las organizaciones y las relaciones de consenso o represión en la sociedad. Un orden político está débilmente institucionalizado cuando no todos los ciudadanos conocen, aceptan o practican un mínimo de reglas de juego institucional.

En este escenario, a Colombia se le ha visto como un país con régimen político débil. En este tipo de regímenes, las estructuras y prácticas de poder político y acción estatal están determinadas por la coyuntura que atraviesan los intereses privados en el momento, lo que asegura incertidumbre e inestabilidad en la capacidad gubernamental, donde lo político no se construye con la diferencia deliberante sino con la negación del otro, y lo público no se constituye a partir de un pacto social para el orden político e institucional, sino con base en la agregación de intereses privados (28).

Ante esta posible situación, que lógicamente afecta el campo de la salud mental, deberían hacerse análisis de su política en un sentido democrático, como lo recomienda Roth, quien muestra dos grandes tendencias para el análisis de políticas: la perspectiva top-down tradicional y la perspectiva democrática:

La perspectiva top-down tradicional, constituye una "herramienta que apunta a ofrecer soluciones de ingeniería social e institucional [...] para la legitimación del orden dominante”, mientras el análisis desde la perspectiva democrática, permite controvertir el papel del Estado y de las instituciones políticas en su definición de la realidad social, cuestiona el marco normativo y cognitivo instituido dominante [...] y permite reintroducir en el centro de los procesos políticos administrativos una democracia más emancipatoria o libertaria. $(25$, p. 22)

Para el mismo autor, en este contexto entra en juego el ejercicio ético-reflexivo del analista de políticas que “orientado por valores" puede contribuir a una "ciudadanía (más) deliberante.

En términos de invitación a profundizar en el análisis de la política pública en el campo de la salud mental en Colombia, caben preguntas como:

¿Quiénes han sido los actores implicados en la política de salud mental en Colombia?

¿Cuáles sus roles?

¿Cómo ha sido la dinámica de relaciones y tensiones entre los actores implicados?

¿Qué debe considerar la política con respecto a la realidad social, cultural, política y económica del país? ¿Cuál es la concepción o concepciones de salud mental más pertinentes para la realidad colombiana? 
¿Cuáles son los principios éticos que deben guiar una política de salud mental para Colombia?

¿Quiénes deberían participar en su formulación?

¿Cuáles serían los obstáculos para la puesta en marcha de la política?

¿Qué le aportaría la política al presente y el futuro del país?

Para concluir, se hace énfasis en la paradoja en salud mental en el sistema de salud colombiano, incoherencia que pudo ser identificada a partir de la perspectiva de profesionales que desde diversos roles han trabajado en el campo. Es visible la defensa de los derechos humanos con base en la normatividad en salud mental, pero al mismo tiempo una estructura del sistema, igualmente evidente en la legislación, que propende por el rendimiento económico, con falencias en los servicios de salud mental, tanto en el primer nivel de atención como en la rehabilitación. A partir de ello, se considera necesario un análisis ético y político, más que técnico, de la política de salud mental en Colombia, y desde allí propender por una mayor participación social en las políticas de salud mental. En las palabras de uno de los participantes del estudio, se requiere "un pacto social por la salud".

\section{Agradecimientos}

Al Fondo de Apoyo Docente del Centro de Investigación de la Facultad Nacional de Salud Pública, por su aporte financiero a la investigación Tendencias en normatividad, administración e investigación en recursos humanos en salud mental para la atención de niños y adolescentes, Colombia, 2006-2013, realizada entre el 2013 y el 2015, código INV-417-13, a partir de la cual se produjo este artículo. Al Grupo Académico de Salud Mental de Antioquia, por sus memorias del foro "Implicaciones de la ley de salud mental desde las perspectiva de los actores". Y a Colciencias, por su apoyo a la formación por medio de la Convocatoria Doctorados Nacionales 647 de 2014.

\section{Referencias bibliográficas}

1. Organización Mundial de la Salud. Informe sobre la salud en el mundo, 2001: Salud mental: nuevos conocimientos, nuevas esperanzas [Internet]. Ginebra; 2001 [citado 2017 jul. 15]. Disponible en: https://www.paho.org/uru/index.php?option=com_docman\&view=download\&alias=134-informe-salud -mental-oms-2001\&category_slug=publicaciones-salud-mental\&Itemid=307

2. Organización Mundial de la Salud. Declaración de Caracas. Caracas: Organización Panamericana de la Salud [Internet]; 1990 [citado 2017 jul. 15]. Disponible en: https://www.oas.org/dil/esp/declaracion_de_caracas.pdf

3. Caldas de Almeida JM. Estrategias de cooperación técnica de la Organización Panamericana de la Salud en la nueva fase de la reforma de los servicios de salud mental en América Latina y el Caribe. Rev Panam Salud Pública [Internet]. 2005;18(4-5):314-26. Disponible en: http://www.bvs.sa.cr/saludmental/28094.pdf

4. Rodríguez J, editor. La reforma de los servicios de salud mental: 15 años después de la Declaración de Caracas [Internet]. Washington: Organización Panamericana de la Salud; 2007. [citado 2017 jul. 15]. Disponible en: https://www1.paho.org/hq/dmdocuments/2009/Reforma\%20de\%20las\%20servicos\%20de\% 20sald\%20mental.pdf

5. Eaton J, McCay L, Semrau M, et al. Scale up of services for mental health in low-income and middle-income countries. Lancet [Internet]. 2011;378:1592-603. Disponible en: https://www.thelancet.com/pdfs/journals/la ncet/PIIS0140-6736(11)60891-X.pdf

6. Onocko-Campos RT, Furtado JP. Entre a saúde coletiva e a saúde mental: um instrumental metodológico para avaliação da rede de Centros de Atenção Psicossocial (CAPS) do Sistema Único de Saúde. Cadernos de Saúde Pública [Internet]. 2006;22(5):1053-62. Disponible en: https://doi.org/10.1590/S0102-311X200600050001 8 
7. Organización Mundial de la Salud. Plan de acción integral sobre salud mental 2013-2020. Ginebra: Asamblea Mundial de la Salud, 2013 [Internet] [citado 2017 jul. 15]. Disponible en: https://apps.who.int/iris/bitstream /10665/97488/1/9789243506029_spa.pdf

8. Thornicroft G. Most people with mental illness are not treated. Lancet. 2007; 370:807-8. Disponible en: https://w ww.thelancet.com/journals/lancet/article/PIIS0140-6736(07)61392-0/fulltext

9. Ngui EM, Khasakhala L, Ndetei D, et al. Mental disorders, health inequalities and ethics: A global perspective. International Review of Psychiatry [Internet]. 2010;22(3):235-44. Disponible en: https://www.ncbi.nlm.nih.g ov/pmc/articles/PMC2935265/pdf/nihms226164.pdf

10. República de Colombia. Rompecabezas de la memoria ¿Aportes a una comisión de la verdad? Bogotá: Centro de Memoria, Paz y Reconciliación [Internet]. 2014 [citado 2017 jul. 15]. Disponible en: https://centromemoria.gov.co/wp-content/uploads/2015/05/Rompecabezas-de-la-Memoria.-Aportes-a-u na-comisi\%C3\%B3n-de-la-verdad-en-Colombia.pdf

11. Gañán JL. Los muertos de la Ley 100. Prevalencia de la libertad económica sobre el derecho fundamental a la salud: Una razón de su ineficacia. Caso del Plan Obligatorio de Salud del Régimen Contributivo [Internet]. Medellín: Universidad de Antioquia, Facultad de Derecho y Ciencias Políticas; 2013 [citado 2017 jul. 15]. Disponible en: https://hrlibrary.umn.edu/research/colombia/Los\%20muertos\%20de\%20la\%20Ley\%20100-Libro.pdf

12. Restrepo MH. Biopolítica: elementos para un análisis crítico sobre la salud mental pública en la Colombia contemporánea. Rev Gerenc Polit Salud [Internet]. 2012;11(23):39-55. Disponible en: https://revistas.javeria na.edu.co/index.php/gerepolsal/article/view/4457/3432

13. República de Colombia. Ministerio de Salud. Resolución 2358 de 1998: por la cual se adopta la Política Nacional de Salud Mental [Internet]. Bogotá: El Ministerio; 1998 [citado 2017 jul. 15]. Disponible en: https://docs.sup ersalud.gov.co/PortalWeb/Juridica/OtraNormativa/R2358_98.pdf

14. República de Colombia. Ley 1616 del 21 de enero de 2013. Por medio de la cual se expide la Ley de salud mental y se dictan otras disposiciones [Internet]. Bogotá: Congreso de la República. 2013 [citado 2017 jul. 15]. Disponible en: https://www.presidencia.gov.co/Normativa/Leyes/Documents/2013/LEY\%201616\%20DEL \%2021\%20DE\%20ENERO\%20DE\%202013.pdf

15. Asociación Colombiana de Psiquiatría. Política Nacional del Campo de la Salud Mental. Documento - Propuesta para discusión y acuerdos [Internet]. Bogotá: Ministerio de la Protección Social. 2007 [citado 2017 jul. 15]. Disponible en: http://psiquiatria.org.co/web/wp-content/uploads/2013/05/Pol\%C3\%ADtica-Nacional-delCampo-de-la-Salud-Mental-versi\%C3\%B3n-final-2.pdf

16. República de Colombia. Ministerio de Salud y Protección Social y la Organización Panamericana de la Salud (OPS/OMS). Documento propuesta de ajuste de la política nacional de salud mental para Colombia. 2014 [Internet]. Bogotá [citado 2017 jul. 15]. Disponible en: https://psiquiatria.org.co/web/wp-content/uploads/2014/07/Documento-propuesta-Pol\%C3\%ADticaNacional-de-Salud-Mental-Colombia-2014-Conflicto-de-codificaci\%C3\%B3n-Unicode.pdf

17. Hoyos G. Hermenéutica práctica: Habermas vs Gadamer. FOLIOS, Revista de la Facultad de Humanidades, Universidad Pedagógica [Internet]. 2003;17:1-17. Disponible en: http://www.pedagogica.edu.co/storage/folio s/articulos/folios17_06arti.pdf

18. Flick U. Introducción a la investigación cualitativa. Madrid: Ediciones Morata; 2007.

19. Anselm S, Corbin J. Bases de la investigación cualitativa: técnicas y procedimientos para desarrollar la teoría fundamentada. Segunda edición. Medellín: Universidad de Antioquia; 2002.

20. República de Colombia. Ministerio de Salud. Resolución 8430 de octubre 4 de 1993. Normas científicas, técnicas y administrativas para la investigación en salud [Internet]. 1993; 4 oct. [citado 2017 jul. 15]. Disponible en: https://www.minsalud.gov.co/sites/rid/Lists/BibliotecaDigital/RIDE/DE/DIJ/RESOLUCION-8430-D E-1993.PDF 
21. Declaración de Helsinki de la Asociación Médica Mundial, Principios éticos para las investigaciones médicas en seres humanos. Dcto 59a Asamblea General [Internet]. 2008; oct. [citado 2017 jul. 15]. Disponible en: https://www.wma.net/es/policies-post/declaracion-de-helsinki-de-la-amm-principios-eticos-para-las-inves tigaciones-medicas-en-seres-humanos/

22. República de Colombia. Ministerio de Salud y Protección Social. Ley 100 de 1993 Por la cual se establece el Sistema General de Seguridad Social en Colombia [Internet]. Bogotá: El Ministerio; 1993. Disponible en: https://www .secretariasenado.gov.co/senado/basedoc/ley_0100_1993.html

23. República de Colombia. Ministerio de Salud y Protección Social. Ley 1122 de 2007 Por la cual se hacen algunas modificaciones en el Sistema General de Seguridad Social en Salud y se dictan otras disposiciones [Internet]. Bogotá: El Ministerio; 2007 [citado 2017 jul. 15]. Disponible en: https://www.minsalud.gov.co/sites/rid/List s/BibliotecaDigital/RIDE/DE/DIJ/ley-1122-de-2007.pdf

24. República de Colombia. Ministerio de Salud y Protección Social. Ley 1438 de 2011 Por la cual se reforma el Sistema General de Seguridad Social en Colombia y se dictan otras disposiciones [Internet]. Bogotá: El Ministerio; 2011 [citado 2017 jul. 15]. Disponible en: https://www.minsalud.gov.co/Normatividad_Nuevo/LEY\%201438\%20 DE\%202011.pdf

25. Roth DA. Las políticas y sus principales enfoques analíticos. En: Roth DA, editor. Enfoques para el análisis de políticas públicas. Bogotá: Editorial Universidad Nacional de Colombia; 2010. p. 17-65.

26. Lahera E. Encuentros y desencuentros entre política y políticas públicas. En: Franco R, Lanzaro J, coordinadores. Política y políticas públicas en los procesos de reforma de América Latina. Buenos Aires: Miño y Dávila Editores. 2006. p. 75-99. Disponible en: https://pastoralurbana.files.wordpress.com/2013/01/flacso.pdf

27. Stone D. Policy paradox: the art of political decision making. New York: Norton; 2002.

28. Medellín P. La política y las políticas públicas en América Latina. Una propuesta para abordar las políticas públicas en regímenes de 'obediencias endebles'. En: Cepal, Flacso. Política y políticas públicas en los procesos de reforma de América Latina [Internet]. Buenos Aires: Miño y Dávila; 2006 [citado 2017 jul. 15]; p. 101-44. Disponible en: https://repositorio.cepal.org/bitstream/handle/11362/2810/S301441F825P_es.pdf;jsessionid $=\mathrm{D} 5 \mathrm{~B} 21 \mathrm{~A} 0792 \mathrm{E} 9 \mathrm{~A} 3 \mathrm{C} 8 \mathrm{~A} 9052 \mathrm{EE} 511 \mathrm{E} 282 \mathrm{~F} 6$ ? squence $=1$

\section{Licencia Creative Commons CC BY 4.0}

Para citar este artículo: Hernández Holguín DM, Sanmartín-Rueda CF. La paradoja de la salud mental en Colombia: entre los derechos humanos, la primacía de lo administrativo y el estigma. Rev. Gerenc. Polit. Salud. 2018;17(35). https://www.doi.org/10.11144/Javeriana.rgps 17-35.psmc 
Dora María Hernández Holguín, et al. La paradoja de la salud mental en Colombia 